\title{
CLOSING THE GAP: THE MATURING OF QUALITY ASSURANCE IN AUSTRALIAN UNIVERSITY LIBRARIES
}

\section{Karen Tang}

A benchmarking review of the quality assurance practices of the libraries of the Australian Technology Network conducted in 2006 revealed exemplars of best practice, but also sector-wide gaps. A follow-up review in 2010 indicated the best practices that remain relevant. While some gaps persist, there has been improvement across the libraries and the development of greater "quality maturity".

Karen Tang, Curtin University Library, GPO Box U1987, Perth WA 6845.

Email: k.tang@curtin.edu.au

\section{INTRODUCTION}

In 2005 the Libraries of the Australian Technology Network (LATN) initiated a project to review current practice in quality assurance across its member libraries. The aims of the study were to describe participants' practices, draw out examples of best practice, and identify gaps and possible areas for improvement.

Participating libraries comprised:

- Auckland University of Technology,

- Curtin University of Technology in Perth

- Queensland University of Technology in Brisbane

- Royal Melbourne Institute of Technology University

- University of South Australia in Adelaide and

- University of Technology, Sydney. 
In 2009/2010 the participants were re-surveyed to investigate whether changes had occurred in the intervening period, including what improvements had been made and where there were still gaps.

In the interim all but one of the institutions with which the libraries were affiliated had undergone a quality assurance audit by the Australian Universities Quality Agency (AUQA) and had witnessed the increased focus on quality assurance and rankings which Yerbury included among her "top 21 changes and challenges for universities" over the decade to 2005 (Yerbury in Williamson 2006: 549).

This paper aims to describe the features of quality assurance found among the LATN participants in 2005/6, to outline the changes which have occurred since and some of the factors which may have contributed to these changes. It aims to set the libraries' quality assurance development or the "meta-quality" of the libraries within the model of quality maturity developed in the United Kingdom by Wilson and Town (2006).

While the number of libraries which were included in the study is small, it is considered that most libraries will find value in the best practices identified for assessing their current state of quality maturity, and that the model provided by Wilson and Town will suggest to them directions in which they might usefully go. The experience of the LATN group since 2005 are likely to be reflective of broader changes across the Australian higher education sector and serve to illustrate how quality maturity can and does change over time.

\section{LITERATURE REVIEW}

While rankings and ranking schemes abound for higher education, an accepted scheme against which LATN could evaluate members' performance on quality assurance was lacking in 2005/6, and still absent in 2009/10. Some best practices and benchmarks had been identified in the work of Wilson and Pitman (2000) and McKinnon, Walker and Davis (2000). But the review of the latter by Garlick and Pryor (2004) and a survey of university libraries undertaken in 2002 (CAUL 2002) indicated limited application of the benchmarks.

In the United Kingdom, Wilson and Town (2006) confirmed that "there is much in the literature ... about frameworks for measuring the quality of a product, process or service but no framework for measuring the quality of the quality-process" (p. 76).

Wilson (2006: 86) coined the term "meta-quality" for an assessment of "the quality of your [library's] quality culture". She argued that: 
"meta-quality is not merely the latest method of measuring service quality. Instead it is concerned with the culture of the library, specifically the quality culture... Libraries today need the same attitude as successful companies: a vision of quality as central to the long-term success of a library; a culture that supports striving for continuous improvement; and strategic systems that are able to convert the desire for improving quality into practice on the ground".

To assist libraries to assess and improve their meta-quality, since 2006 Wilson and Town have been developing what they have called a Library Quality Maturity Model measuring a library's quality maturity on a five-step scale, from Level 1 - Initial, to Level 5 - Optimising. The model is based on the sole framework they found for measuring quality culture - the Capability Maturity Model developed by the Software Engineering Institute as a method of judging the quality of the software processes of an organisation (Wilson, 2006: 86).

While the initial LATN survey pre-dated the publication of Wilson and Town's work and thus was not based on their quality maturity criteria, the levels in the quality maturity model have the potential to provide a valuable roadmap, indicating not only where LATN libraries are in their quality maturity, but where they - and other libraries - might go.

\section{METHODOLOGY}

In 2005/6 the LATN reviewers undertook a review of member libraries' websites. In addition preliminary information was gathered via a questionnaire completed by a nominee from each member library, and follow-up in-person interviews with each nominee and the University Librarian of each institution. The 2005 questionnaire, largely unchanged, was re-distributed during the summer of 2009/10. Due to restructuring and staffing changes at most libraries, with one exception the questionnaire was completed by a different nominee in 2009/10 than 2005. The 2009/10 questionnaire also asked respondents to highlight changes made since 2005/6. No interviews or site visits were conducted in 2009/10.

For the purposes of the reviews, a broad view of "quality assurance" was taken including planning and performance measurement; use of frameworks, charters and service level agreements; benchmarking and evaluation. Responsibilities, training and communication in relation to quality were considered, together with the extent to which it could be said that quality was embedded in the culture of the library.

The reviewers heeded Garlick and Pryor's cautions against a "'onesize fits all' top-down approach" (2004: viii). They instead attempted to identify some principles and exemplars of best practice in quality assurance, leaving it to each member library to determine whether a particular practice was appropriate in its context.

"Best practice" was defined as: 
The pursuit of world class performance. It is the way in which the most successful organisations manage and organise their operations. It is a moving target. As the leading organisations continue to improve the 'best practice' goalposts are constantly moving. The concept of continuous improvement is integral to the achievement of best practice.

1994 Australian Best Practice Demonstration Program definition, quoted in Wilson, Pitman, and Trahn (1999: 59)

Where no exemplar of best practice could be found amongst the participating libraries by the reviewers, this was identified as a sectorwide "gap".

Four reports were published:

- An interim, descriptive report summarizing 2005/2006 quality assurance practices (Tang \& Levinge, 2006a).

- A final, evaluative report of the 2005/2006 situation, providing principles for quality assurance processes, criteria for and examples of best practice amongst members, and sector-wide "gaps" which ought to be addressed (Tang \& Levinge, 2006b).

- A descriptive report based on the $2009 / 10$ questionnaire (Coen, 2010) and

- An analysis of the results of the 2005/6 and 2009/2010 reviews (Tang, 2010).

[Hereafter, for simplicity the studies will be referred to as the 2006 and 2010 reviews, although in both cases they commenced a year earlier.]

All four reports are available through the LATN Collaborative Online Workshop (COW) [http://www.latn.edu.au/]. The COW also provides access to many of the documents referred to in the reports.

\section{FINDINGS}

As noted above, the 2006 reviewers' determination of "best practice" was their own, guided by the work of Wilson and Pitman (2000), McKinnon, Walker and Davis (2000) and Wilson and Town (2006) together with their own observations of the participating libraries. The 2010 reviewers did not identify any need to change these standards.

The best practices identified by the reviewers are listed in Table 1. This Table also indicates the extent to which the reviewers identified the best practices to be in place or have exemplars in 2005 and subsequently in 2010, together with an indication as to whether there had been an increase/improvement in the adoption of the practice in the interim.

The following section elaborates on the reviewers' rationale in selecting the best practices and some of the changes observed. 


\section{Quality Assurance framework and responsibility}

The reviewers considered that a functioning and well-maintained quality framework introduced an explicit and transparent model of systematic quality assurance to a library.

The publishing of a schedule of planning and performance measurement activities which would occur in each year of the quality cycle was considered to indicate a commitment to them, making them explicit and transparent to all and with practical benefits in terms of scheduling and administrative procedures.

Responsibility for quality could be centralised (with a designated quality officer), within a manager's portfolio, or devolved where all managers were responsible for implementing quality within the library's framework. No model was considered to be perfect, with a different model being more appropriate depending on the library's level of quality maturity. The reviewers considered that a centralised model at the early stages of the development of a quality assurance program gave the development and implementation of the library's quality framework a dedicated driver. Once the quality program was in place, more devolution could take place.

Between the 2006 and 2010 surveys the reviewers found a doubling in the use of quality assurance frameworks and the sector-wide gap in the use of an associated schedule of activities had been overcome in two libraries. Half the libraries had created new quality officer positions while one had moved on to a more devolved model.

\section{Planning}

The reviewers believed that a medium-term planning outlook, i.e. beyond one year was necessary, including explicit longer-term objectives and/or a shared vision of direction and future priorities.

Alignment with higher level university plans needed to be genuine, not simply template-driven form-filling.

Best practice also required a cascading down of plans and planning, aligning plans for sections, units and committees of the library and genuine individual work planning in which the individual and their supervisor considered and recognized the individual's contribution to the library's broad objectives / planning initiatives or the achievement of performance indicators/targets.

Medium-term plans were more widespread in 2010 than in 2006, with all but two libraries having at least a 3-year plan. Compared with 2006, most libraries appeared to be striving for genuine alignment with higher level plans. Identified as a sector-wide gap in 2006, individual work planning was more widespread by 2010, with all but one library mentioning that individual work planning and performance review was now in place.

The reviewers agreed strongly with the arguments of McKinnon, Walker and Davis (2000: 118) and Wilson and Pitman (2000: 3) 
that staff "ownership" of their library's plan is vital and looked for a variety of means being open to staff to contribute their ideas in the planning process - directly (through attendance at planning activities) or indirectly (through staff input of ideas in advance of planning session(s)). They also agreed with McKinnon, Walker and Davis (2000: 118) who argued that client or stakeholder involvement in planning was desirable to develop effective partnerships and support.

Growth in library staff involvement in planning was evident between the 2006 and 2010 surveys, but this was not true of client or stakeholder involvement.

The reviewers argued that communication of library plans was vital so that library staff could feel some ownership of their library's plan and for greater awareness amongst clients and stakeholders. Some effective communication methods to library staff were noted in 2006 but targeted, planned, and consistent communication of library plans to clients and key stakeholders was considered to be a sector-wide gap.

By 2010 libraries were paying greater attention to the communication of their plans to their staff and, to some extent, clients.

Monitoring and reporting of progress on plans was considered to be best-practice and in place in all libraries with regard to their annual plans. However monitoring and assessing progress toward the libraries' medium-term objectives was considered a gap across the sector in 2006 which had been overcome by only one library in 2010. While some libraries took time at the end of the medium-term plan to assess what had been achieved, during the life of the plan libraries seemed to assume that achievement of annual plans was progressing the library toward the achievement of its medium-term goals.

\section{Performance measurement}

The reviewers noted that on-going measurement of performance in key areas is part of most models of library quality assurance. In 2006 they found no shortage of performance measurement in the participating libraries but the extent to which the measurement was systematic and targeted, with results consistently analysed and acted upon, was questionable. One library was commended for its use of a performance framework and dedicated performance meetings but by 2010 no other libraries had adopted such a framework. The libraries were however moving or had moved toward more targeted and co-ordinated approaches to performance measurement. All libraries reported having strategic or key performance indicators aligned with their Library or University Plan and new systems for their management had been introduced. More libraries noted that their performance results were reported to, and considered by, a library management group and provided to library staff and clients through intranets, websites and annual report type publications 
The reviewers considered that valuable albeit less strategic practices in performance management included a client charter with clearly defined performance standards and monitoring and reporting against those standards; periodic conduct of client and library staff satisfaction/perception surveys with mechanisms to collect feedback between surveys, and mechanisms for quick assessments when necessary. With the exception of the latter most of the participants made some use of all of these in both 2006 and 2010.

On the contrary in 2006 the reviewers found that few respondents had been involved in impact or outcomes measurement activities although this was a growing desire amongst libraries internationally, albeit difficult to achieve (Missingham, 2005: 156; Payne \& Conyers, 2005: 1). By 2010 this gap had still not been addressed.

\section{Benchmarking and external evaluation/assessment}

The use of benchmarking was identified as a gap in the 2006 study. In 2010, all but one library claimed to undertake some form of performance benchmarking, but there was little evidence of libraries undertaking systematic, targeted comparisons and attempting to learn from or emulate the top performers. One library had recently developed a Benchmarking Framework and aimed to move from comparison to true benchmarking over the life of its new Library Plan.

A notable growth in external reviews of libraries was observed between 2006 and 2010, with external reviews other than AUQA visits either included as part of the routine planning and performance measurement process, or initiated/participated in at more random intervals.

\section{Embedding of quality assurance in the library's culture}

Consistent with Wilson (2006: 86), the reviewers considered that best practice involved the development of a culture of quality across the library, not just at the management level. In 2006 most respondents considered that quality assurance was reasonably embedded at the senior and management levels but were less confident regarding the remainder of library staff. In 2010 most libraries reported that quality assurance had become more embedded into their culture since 2006. An objective assessment could not be made by the reviewers as consultation with library staff was beyond the scope of the studies.

Systematic training for quality is a key criterion of Wilson and Town's quality maturity model. Other than in preparation for AUQA audits, the reviewers did not find any evidence of specific training of library staff in quality in either 2006 or 2010 and considered this to be an important sector wide gap.

\section{AND 2010 RESULTS COMPARED}

In summary, comparing 2010 with 2006, the reviewers noted improvement in several areas: 
- More designated quality officers had been appointed and there was more evidence of focussed, co-ordinated, systematic activity.

- There was greater use of quality/planning/performance frameworks and a longer-term outlook in planning. Initiatives were more commonly scoped and monitored with a project management framework sometimes applied.

- Use of strategic performance indicators was more widespread with some new systems adopted for managing the plethora of statistics libraries collect.

- Individual work planning was more widespread, taking quality concepts such as ADRI and continuous improvement beyond library management.

- Engagement of library staff with quality activities and communication of results to clients had also seen improvement.

In both 2006 and 2010 participants were asked to rate their library's quality assurance on a five point scale. In both years all rated themselves 3 or 4 or some fraction in between. In 2010 one library allocated itself a lower score than in 2006, three others a higher score, while two others allocated the same score.

\section{IMPROVEMENT EXPLANATIONS}

The reviewers did not seek an explanation from respondents as to why particular practices had altered. However some explanations were offered or can be deduced.

Some of the improvements were attributed by respondents to what they learnt from other member libraries through the 2006 project. For example Library A adopted and adapted Library B's "scorecard on a page" while Library B introduced scoping documents for initiatives based on Library A's templates. Such developments were exactly what the sponsors of the surveys and the reviewers had hoped for!

Two libraries noted that the appointment of a dedicated position for their planning and quality processes had led to improvements and the spread of a quality culture through and beyond the library's management group.

Other improvements were attributed to developments and requirements within the university of which the library formed part and to university preparations for AUQA audits. For example one library reported implementing changes as a result of a university-wide business improvement process review. Several noted changes in university requirements for strategic planning and particularly individual work planning and performance review. Some libraries attributed an increased awareness of quality language and processes by library staff to university preparations for AUQA audits and staff involvement in individual planning. The greater focus on the communication of plans to staff, clients and stakeholders was specifically attributed to university preparations for AUQA audits. 
Such explanations are not surprising given the changes between 2006 and 2010 in the environment in which the libraries' parent universities were operating.

The period saw a continuation in the growing competition between Australian universities and other higher education providers (national and international), and between Australian universities themselves (Yerbery in Williamson, 2006: 549). This competition was coupled with a desire among many universities, including members of the Australian Technology Network, to be recognised not only or primarily for their teaching but for their research. To this can be added the Australian government's commitment to making comparative quantitative information on education publicly available, through sites such as the "My School" website and the planned "My University" site. In such an environment positive performance data, comparisons, benchmarking and rankings are of vital importance, to identify and/ or publicise how the university (and its component parts such as its library) is performing vis-a-vis competitors.

During the period all but one of the parent universities of the members of LATN underwent at least one AUQA quality assurance audit. For their audits, universities were required to submit portfolios documenting their approach to quality assurance and during their visits, the AUQA auditors interviewed large numbers of staff and students. Preparations for such audits focussed universities on their quality assurance documentation, processes, training and communication to staff, students and stakeholders.

More extensive use of individual work planning may be part of university planning improvements in preparation for AUQA audits or may be attributable to the 2007 government introduction of Higher Education Workplace Relations (HEWR) Requirements, which required compliance as a condition of funding increases. One of the HEWR Requirements was the existence of performance management schemes for university staff.

\section{QUALITY MATURITY}

Looking at the findings of the LATN studies in a broader, international context, the improvements noted between 2006 and 2010 can be seen as a "maturing" of quality assurance on the continuum of quality levels defined by Wilson and Town (2006: 77-78). Table 1 below provides an overview of Wilson and Town's "Quality Maturity Model" and the criteria they identified to place libraries at various levels.

On the criteria related to quality processes, whereas it could have been argued in 2006 that most of the libraries were at maturity level 1 (with quality being achieved in an ad hoc way reliant on individual effort and heroics), by 2010 this no longer seemed to be the case. The appointment of quality officers and adoption of quality-related frameworks suggest progress of all the libraries to at least level 2 (with quality policies and procedures to implement these policies established). The greater availability and monitoring of medium-term as well as annual plans might indicate movement to level 3 (which 
requires that there is a defined, documented organisational strategy) though it is doubtful that any of the participants could claim that all their work processes derive from this strategy (level 3), or that quality measures are part of every documented work process (level 4) or that the entire organisation is focussed on continuous improvement in every service, product and process (level 5).

On the quality measurement criteria, compared with 2006, by 2010 there were more effective management processes to avoid customer satisfaction being reactive and unpredictable (level 1), with the libraries better positioned to repeat earlier success in customer satisfaction (level 2). The more widespread use of strategic performance indicators, new systems adopted for managing statistics, and monitoring of performance against client charters/ service standards indicate libraries were using quality measurements to evaluate products and services, implementing changes and improvements based on these measures and setting quantitative goals for quality and customer satisfaction (level 4). They were therefore better able to identify weaknesses, the means to strengthen processes and proactively prevent problems (level 5).

The greater use of individual work planning and performance monitoring noted in 2010 suggests there was greater organisationwide understanding of the activities, roles and responsibilities of each member of the organisation and how they fit into the organisational structure (level 3) and all staff were being encouraged to continuously improve themselves and their work (level 5).

Only in regard to the criteria for training in quality do the participating libraries seem not to have progressed between 2006 and 2010. Specific training in quality was notably absent from the libraries in both 2006 and 2010 - at best it could be categorised as ad hoc and reactive (level 1). There was no mention of the training needs assessments, programme provision and measurement or preparation of staff for future organisational requirements associated with higher quality maturity.

An in-depth analysis of the quality maturation of the LATN libraries in relation to responsibility for quality assurance, the use of frameworks and individual work planning and training for quality is available in Tang (2011).

\section{CONCLUSION}

The 2006 and 2010 LATN benchmarking of quality assurance practices across member libraries has enabled participants to gain an understanding of the strengths and weaknesses of their own quality assurance arrangements, to establish what constitutes best practice in this field, and to identify and learn from exemplars of best practice amongst their colleagues. The result has been quality assurance improvement and greater "quality maturity".

Wilson and Town (2006: 79-80) argue that benchmarking can have a long-term effect on library and information service quality, but that 
this effect may only occur in libraries with a more mature quality approach. As each of the libraries of the LATN group achieves a greater level of quality maturity, it is likely that future benchmarking of their quality assurance processes will prove even more worthwhile.

\section{REFERENCES}

CAUL Best Practice Group. 2002. Preliminary survey on McKinnon Benchmarks. http://www.caul.edu.au/surveys/ mckinnon2002.doc

Coen, Marilyn. 2010 Quality assurance within ATN Libraries: a LATN Benchmarking Project. Descriptive report. http://www.latn.edu.au/

Garlick, Steve \& Geoff Pryor. 2004. Benchmarking the University: Learning about improvement. Canberra: Higher Education Division, Department of Education, Science and Training. http://www.dest. gov.au/sectors/higher_education/publications_resources/profiles/ benchmarking_the_university.htm

McKinnon, K. R., S. H. Walker \& D. Davis. 2000. Benchmarking: a manual for Australian universities. Canberra: Higher Education Division, Department of Education, Training \& Youth Affairs.

Missingham, Roxanne. 2005. Libraries and economic value: A Review of recent studies. Performance Measurement and Metrics 6. (3): 142-158.

Payne, Philip \& Angela Conyers. 2005. Measuring the Impact of Higher Education Libraries: The LIRG/SCONUL Impact Implementation Initiative. Library and Information Research 29 (91). http://www.cilip.org.uk/specialinterestgroups/bysubject/research/ publications/journal/archive/lir91/

Tang, Karen. 2010. Quality assurance within ATN Libraries: a LATN Benchmarking Project. Analysis of the results of the 2006 and 2010 LATN Reviews. http://www.latn.edu.au/

Tang, Karen \& Leanne Levinge. 2006a. Quality assurance within ATN libraries: a LATN Benchmarking Project. Interim report. http:// www.latn.edu.au/

Tang, Karen \& Leanne Levinge. 2006b. Quality assurance within ATN libraries: a LATN Benchmarking Project. Evaluative report. http://www.latn.edu.au/

Tang, Karen. 2011. Quality assurance improvements in Australian University Libraries. Paper presented at 9th Northumbria International Conference on Performance Measurement in Libraries and Information Services, August 2011, York, United Kingdom. Publication in process.

Williamson, Vicki. 2006. Surviving change and growing the profession together. Library Management. 27(8): 58-61 
Wilson, Anne \& Leanne Pitman. 2000. Best practice handbook for Australian university libraries. Canberra: Evaluations \& Investigations Programme, Higher Education Division, Department of Education, Training \& Youth Affairs.

Wilson, Anne, Leanne Pitman \& Isabella Trahn. 1999. Guidelines for the application of best practice in Australian university libraries: Intranational and international Benchmarks. Canberra: Evaluations \& Investigations Programme, Higher Education Division, Department of Education, Training \& Youth Affairs.

Wilson, Frankie \& J. Stephen Town. 2006. Benchmarking and library quality maturity. Performance Measurement and Metrics. 7(2): 75-82

Wilson, Frankie. (2007) The Quality maturity model: The story so far. 7th Northumbria International Conference on Performance Measurement in Libraries and Information Services, "Measuring library performance and organizational effectiveness: From research to practice", 13 - 16 August 2007, IFLA Statistics and Evaluation Section, Spier Hotel and Conference Centre, Stellenbosch, South Africa. 


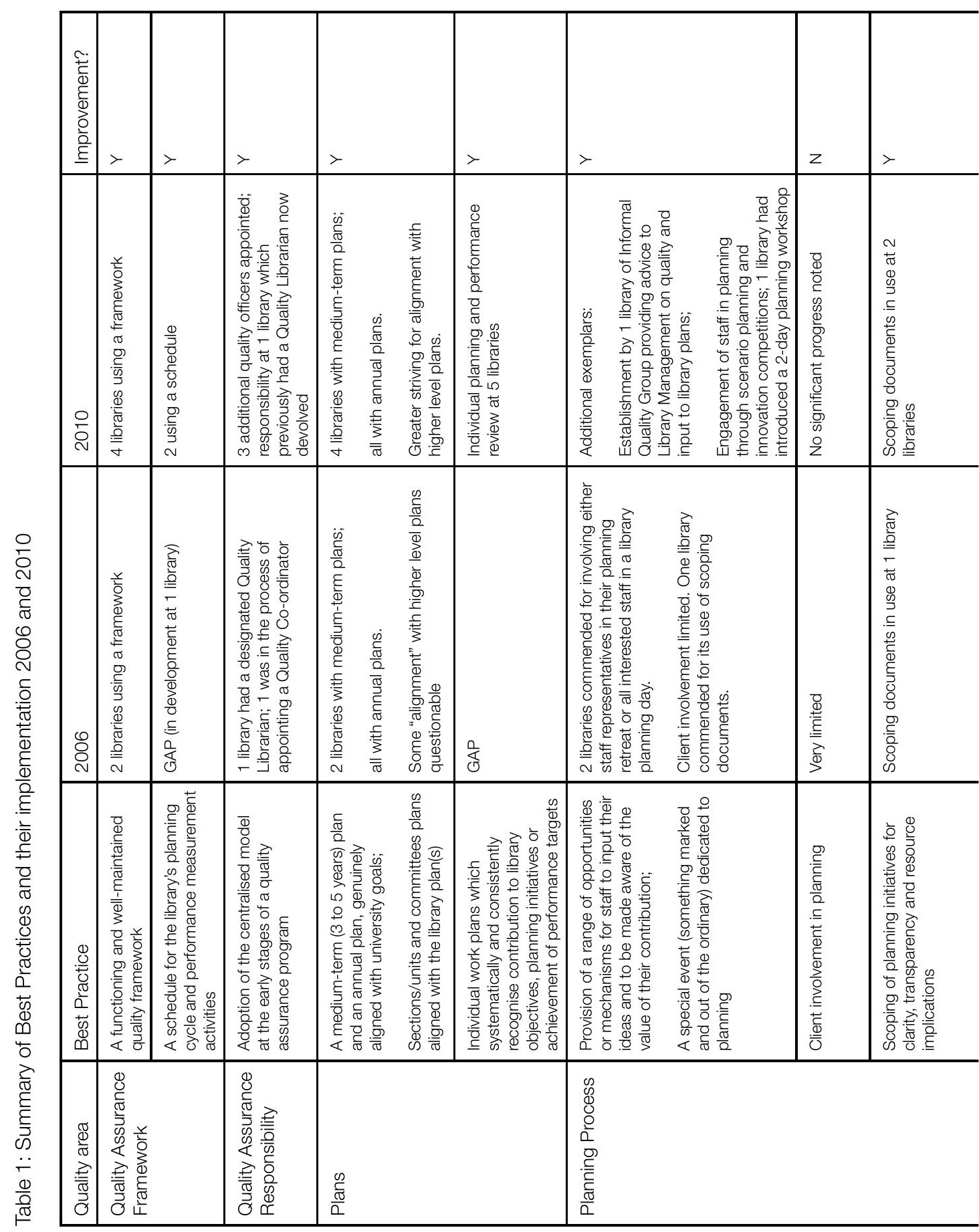




\begin{tabular}{|c|c|c|c|c|c|c|c|c|}
\hline$>$ & $>$ & \multicolumn{2}{|l|}{$>$} & z & $z$ & $>$ & $>$ & $>$ \\
\hline 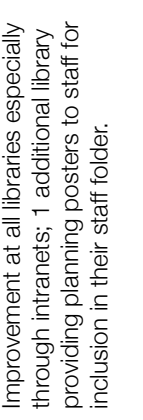 & 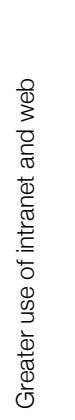 & \multicolumn{2}{|c|}{ 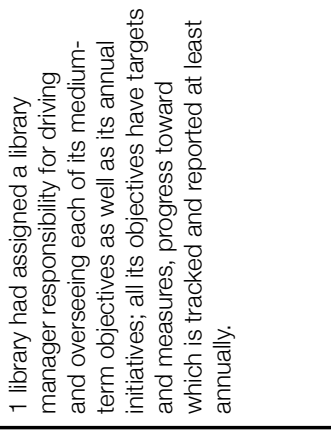 } & 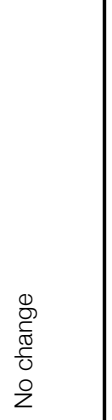 & 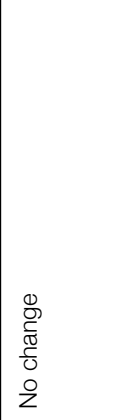 & 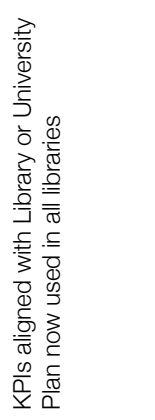 & 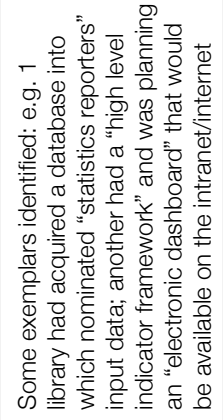 & 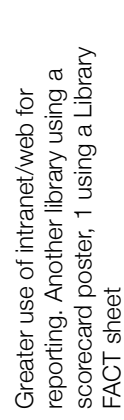 \\
\hline 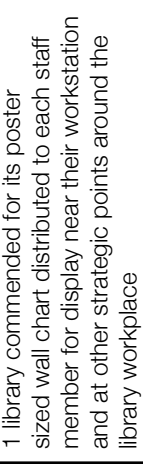 & $\frac{0}{8}$ & 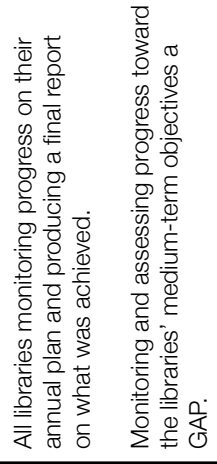 & 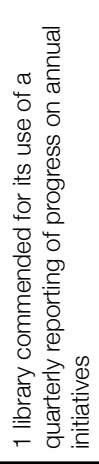 & 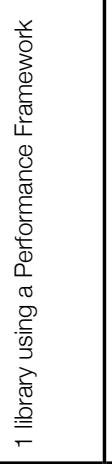 & 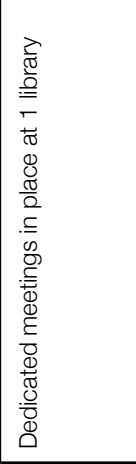 & 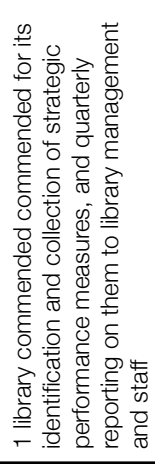 & 莨 & 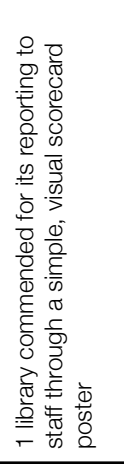 \\
\hline 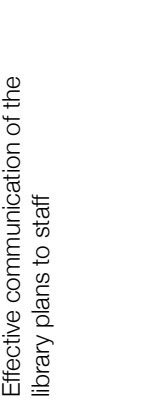 & 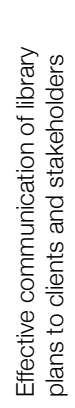 & 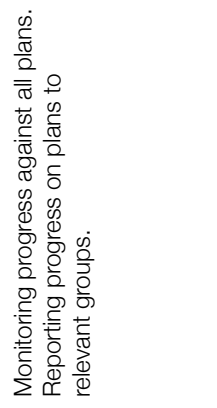 & & 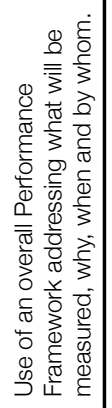 & 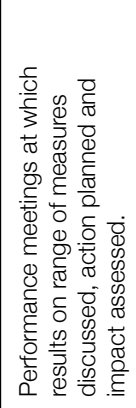 & 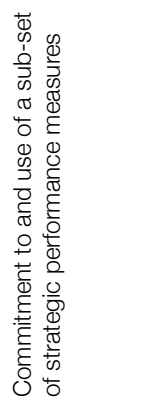 & 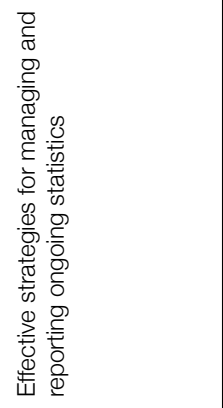 & 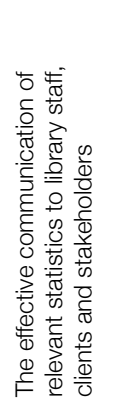 \\
\hline 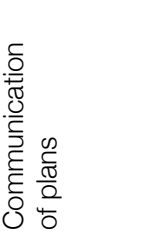 & & 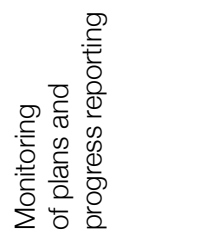 & & 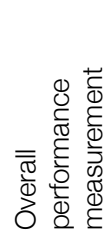 & & & 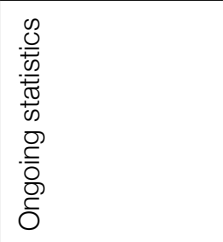 & \\
\hline
\end{tabular}




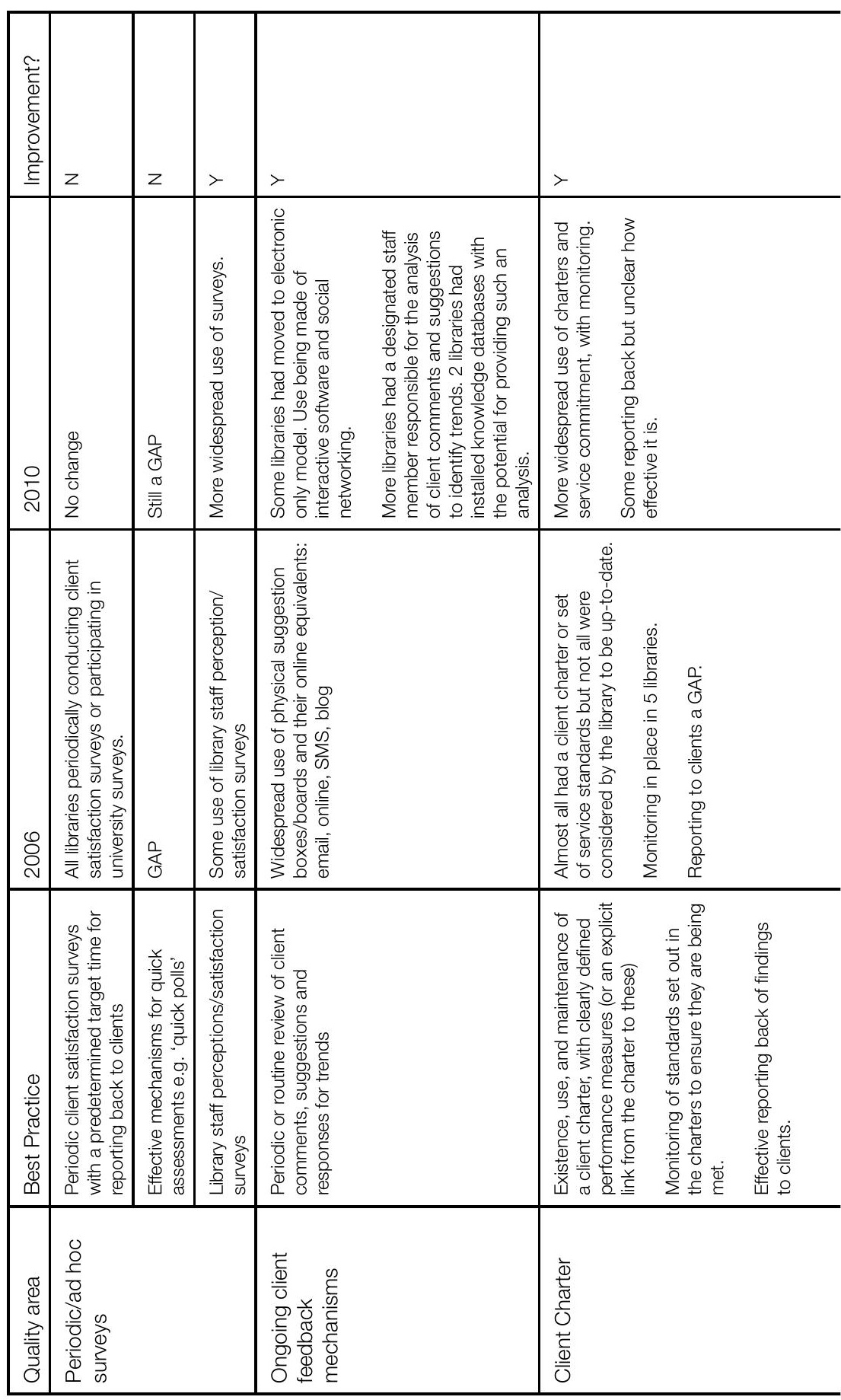




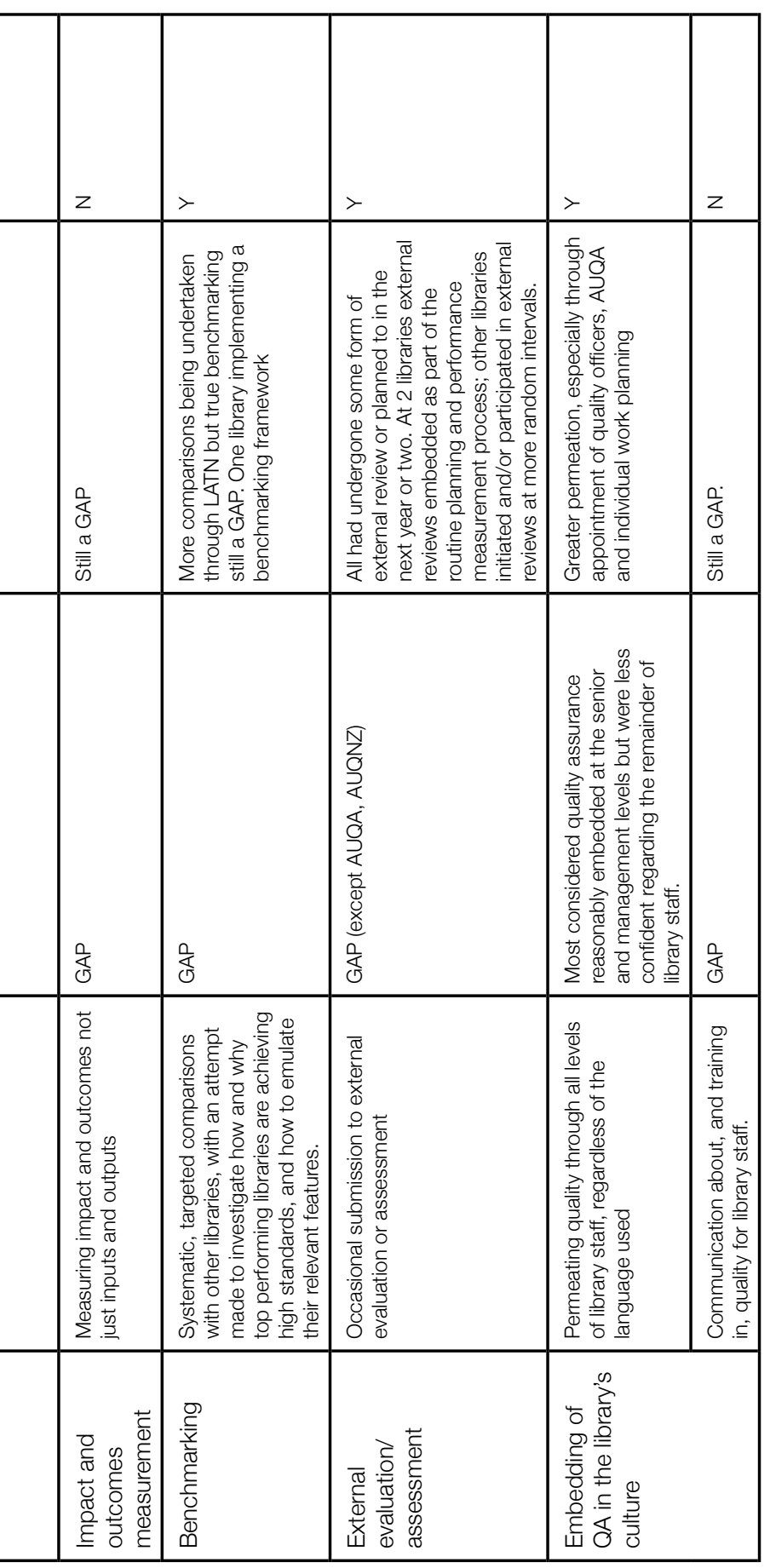


Table 2: Wilson and Town (2006) Quality Maturity Levels

Level 1 - initial. The quality management process is ad hoc, and occasionally even chaotic. Few processes are defined, and success depends on individual effort and heroics:

- quality is achieved in an ad hoc way;

- customer satisfaction is reactive and unpredictable;

- $\quad$ quality depends on the capabilities of individuals, and varies with their innate skills, knowledge and motivations; and

- $\quad$ training for quality is ad hoc and reactive to an ability to undertake a specific task adequately.

Level 2 - repeatable. Basic quality management processes are established. The necessary management processes are in place to repeat earlier quality levels:

- quality policies, and procedures to implement these policies, are established;

- there are effective management processes to allow the organisation to repeat earlier success in customer satisfaction;

- $\quad$ such management processes are practised, documented, enforced, trained, measured, and able to improve; and

- $\quad$ training for quality is provided as a programme of training for specific work tasks, and/or is reactive to events.

Level 3 - defined. The quality processes are documented and standardised. All work derives from the organisational strategy:

- there is a defined, documented organisational strategy, from which all work processes are derived;

- $\quad$ there is an organisation-wide understanding of the activities, roles, and responsibilities of each member of the organisation, and how they fit into the organisational strategy; and

- training for quality is a cycle of training needs assessment and programme provision.

Level 4 - managed. Detailed measures of the quality process are collected. The quality process is quantitatively understood and controlled:

- $\quad$ quality measures are part of every documented work process;

- these measurements form the basis for evaluating products and processes;

- changes are implemented to improve the quality of services, products and processes; 
- the organisation sets quantitative goals for quality and customer satisfaction; and

- training for quality is a cycle of training needs assessment, programme provision, and measurement of the effectiveness of the programme.

Level 5 - optimising. Continuous quality improvement is enabled by quantitative feedback and from piloting innovative ideas:

- the entire organisation is focussed on continuous improvement in every service, product and process;

- $\quad$ all staff are encouraged to continuously improve themselves and their work;

- the organisation is able to identify weaknesses, and the means to strengthen the process, proactively with the goal of preventing problems;

- innovations that exploit the best practices are identified and transferred throughout the organisation; and

- $\quad$ training for quality is focussed on preparing staff for future organisational requirements. 